\title{
Der Einsatz der RehaGoal App durch Menschen mit einer intellektuellen Beeinträchtigung bei der Arbeit zu Hause während der Corona-Pandemie
}

\author{
Funda Ertas ${ }^{1}$, Jasmin Aust ${ }^{1,2}$, Tom Lorenz ${ }^{3}$, Ina Schiering ${ }^{3}$, Katharina Brunner ${ }^{2}$ \\ und Sandra Verena Müller ${ }^{1}$ \\ Ostfalia Hochschule für Angewandte Wissenschaften, Fakultät Soziale Arbeit, Wolfenbüttel \\ DRK-inkluzivo Wolfenbüttel gGmbH, Wolfenbüttel \\ Ostfalia Hochschule für Angewandte Wissenschaften, Fakultät Informatik, Wolfenbüttel
}

\begin{abstract}
Zusammenfassung: Infolge der Corona-Pandemie 2019 und den damit einhergehenden Restriktionen verloren Menschen mit einer intellektuellen Beeinträchtigung - wie andere auch - ihre Praktikumsplätze oder wurden in das Szenario „Arbeit zu Hause“ geschickt. In einer für den ersten Lockdown konzipierten 3-wöchigen Pilotstudie zur Untersuchung einer mobilen Applikation zur Handlungsplanung kochten drei Teilnehmer*innen mit exekutiver Dysfunktion - und daraus resultierenden Schwierigkeiten in der Handlungsplanung - begleitet von Jobcoaches mithilfe der RehaGoal App. Zur Erfassung der Usability dieser App wurde ein teilstandardisierter Fragebogen von den Teilnehmer*innen und ihren Angehörigen beantwortet. Es zeigte sich, dass die Teilnehmer*innen die RehaGoal App gut einsetzen konnten und sich sicher in der Benutzung fühlten. Zudem gaben die Angehörigen an, sich vorstellen zu können, die App für weitere häusliche Aufgaben zu nutzen.
\end{abstract}

Schlüsselwörter: Intellektuelle Beeinträchtigung, exekutive Funktion, assistive Technologie, planerisches Denken, Corona-Pandemie

The use of the RehaGoal app by people with intellectual disabilities working at home during the Coronavirus disease pandemic

Abstract: Because of the Coronavirus disease pandemic and accompanying restrictions, like others people with intellectual disabilities lost their internships or were forced to work at home. As part of a pilot study designed for the first lockdown to investigate a workflow support system, three participants with executive dysfunction and resulting difficulties in action planning were accompanied by job coaches, cooking for three weeks with the help of the RehaGoal app. To record the use of the App, the participants and their relatives filled out a partially standardized questionnaire. The participants felt confident in using the RehaGoal App and hardly had to learn anything for its use. In addition, the relatives stated that they could imagine using the app for other home tasks.

Keywords: intellectual disability, executive function, assistive technology, planning, Coronavirus disease pandemic

\section{Einleitung}

\section{Hintergrund}

Die berufliche Teilhabe ist für Menschen mit intellektuellen und anderen Beeinträchtigungen ,eine wichtige Voraussetzung für ein gleichberechtigtes Miteinander in der Gesellschaft" (Rauch, 2005). Nach Trost (2013) bestimmt die berufliche Teilhabe von Menschen mit intellektueller Beeinträchtigung (IB) „sehr wesentlich den $\mathrm{Zu}-$ gang zu Lebensmöglichkeiten und -chancen [...] und [beeinflusst] die individuelle Lebensbewältigung jedes
Einzelnen ebenso wie seine soziale Integration". Werkstätten für behinderte Menschen sichern für viele Menschen mit Beeinträchtigung nach wie vor den Zugang zur beruflichen Teilhabe. Laut einer Studie der Bundesarbeitsgemeinschaft der überörtlichen Träger der Sozialhilfe (BAGüS) aus dem Jahr 2017 lag die Zahl aller Leistungsberechtigten in Werkstätten für behinderte Menschen bei 275110 Personen. Der Anteil der Menschen mit IB lag dabei bei über $70 \%$ (Bundesarbeitsgemeinschaft der überörtlichen Träger der Sozialhilfe/con sens, 2019). Zudem zeigen aktuelle Studien, dass die Gesamtanzahl der Beschäftigten im Arbeitsbereich von 
Werkstätten für behinderte Menschen zwischen 2008 und 2017 kontinuierlich angestiegen ist (Bundesarbeitsgemeinschaft Werkstätten für behinderte Menschen, 2019; Deutsches Institut für Menschenrechte e.V., 2019).

Die UN-Behindertenrechtskonvention (UN-BRK) stärkt das Recht auf Arbeit für alle Menschen mit Beeinträchtigung. Dies beinhaltet, dass das Arbeitsumfeld selbstbestimmt gewählt werden soll und der Arbeitsmarkt Menschen mit Beeinträchtigung offen zugänglich zu sein hat (Art. 27; Beauftragter der Bundesregierung für die Belange von Menschen mit Behinderungen, 2018). Die Realität sieht für Menschen mit IB nach wie vor anders aus, wie die vorangegangenen Zahlen der Mitarbeiter*innen von Werkstätten für behinderte Menschen zeigen. Gestärkt durch die UN-BRK greifen mehr Menschen mit Beeinträchtigung auf alternative Unterstützungsformen zurück, um ihr Recht auf einen Arbeitsplatz im allgemeinen Arbeitsmarkt in Anspruch zu nehmen. Hierbei werden sie von pädagogischem Fachpersonal begleitet, beispielsweise durch einen Fachdienst zur beruflichen Eingliederung (FBE).

Der FBE bietet Menschen mit körperlichen, intellektuellen, psychischen und Sinnesbehinderungen oder Lernschwierigkeiten die Möglichkeit zu einer beruflichen Rehabilitation. Ziel ist es, für Menschen mit Beeinträchtigung durch verschiedene Praktika einen Platz auf dem allgemeinen Arbeitsmarkt zu finden, an dem sie ihre persönlichen Ressourcen adäquat einsetzen und darüber Akzeptanz und Wertschätzung erfahren können. Dabei bietet der FBE Hilfestellung durch Jobcoaches vor Ort an, die die Teilnehmer*innen in den Betrieben am Arbeitsplatz einarbeiten und begleiten. Bislang ist aus diesem Grund eine individuelle und dadurch personalintensive Betreuung durch Jobcoaches im Rahmen der Teilhabe am Arbeitsleben erforderlich. Grundsätzlich begleitet der FBE Teilnehmer*innen mit sehr unterschiedlichen Beeinträchtigungen im Berufsbildungsbereich, in Unterstützter Beschäftigung, in Integrationspraktika, im Jobcoaching und in Arbeitsassistenz auf dem allgemeinen Arbeitsmarkt. Viele von ihnen leiden unter Defiziten in ihrer exekutiven Funktion (Müller, 2013; Müller \& Klein, 2019) und haben daher beispielsweise Schwierigkeiten bei der Strukturierung von Aufgaben, dem planerischen Denken, der Handlungsorganisation sowie der Selbstorganisation und -regulation. Bereits im Alter zwischen 3 und 5 Jahren beginnt die Manifestation der exekutiven Funktion und verläuft parallel zu der Reifung des Zentralnervensystems, insbesondere der Entwicklung des Frontallappens (Garon, Bryson \& Smith, 2008; Mahone \& Hoffman, 2007; Stuss, 2011). Menschen mit IB zeigen daher schon früh Probleme in ihren exekutiven Funktionen und benötigen daher Unterstützung in ihren alltäglichen und beruflichen Aktivitäten (Memisevic \& Sinanovic, 2013).

\section{Corona-Pandemie-Ausgangslage}

Der durch die Corona-Pandemie bedingte Lockdown hat die Teilnehmer*innen des FBE stark getroffen. Viele verloren ihren Praktikumsplatz, mussten eine Zwangspause einlegen oder konnten geplante Praktika nicht antreten, da zum Beispiel Altenpflegeheime keine externen Besucher*innen erlaubten, Jugendherbergen und der Einzelhandel geschlossen wurden bzw. zu wenig Arbeit für alle Mitarbeiter*innen vorhanden war. Die davon betroffenen Teilnehmer*innen befanden sich - wie viele andere auch - daher zwischen Mitte März und Ende Juni im Workat-Home und wurden mit Arbeitsaufträgen rund um das Thema Arbeit per Mail versorgt. Sie erstellten Referate zu bestimmten Themen, wie zum Beispiel Traumberuf, Klima oder Industrialisierung, oder wurden in Deutsch und Mathe unterrichtet. Zudem gab es individuelle Arbeitsaufträge, wie ein Blumenbeet im Garten zu gestalten oder Aufgaben im Haushalt zu übernehmen.

Während der Zeit im Work-at-Home wurde täglich Kontakt zum Jobcoach gehalten. Bei der Bearbeitung der Aufträge stand dieser für Fragen zur Verfügung oder begleitete die Erarbeitung engmaschig. Die Kommunikation erfolgte über verschiedene mediale Kanäle wie zum Beispiel mittels Telefonaten, Videochats, Fotos oder Fernwartungssoftware. Alle Fortschritte und Ergebnisse der Arbeitsaufträge erhielt der Jobcoach als Fotodokumentation.

\section{Die RehaGoal App}

Im Rahmen der Digitalisierung ergeben sich neue digitale Unterstützungsmöglichkeiten für Menschen mit IB. In ersten Studien wurden bereits technologische Assistenzsysteme im Alltag der Menschen mit IB evaluiert (Cannella-Malone et al., 2006; Sigafoos et al., 2007). Hier zeigte sich, dass sich zum Beispiel beim Einkaufen durch die Anwendung von visuellen Cues und Video-Prompts mithilfe eines digitalen Mediums die Unabhängigkeit verbesserte (Burckley, Tincani \& Guld Fisher, 2014). Auch im beruflichen Kontext wurde bei dieser Zielgruppe mit Prompts auf dem iPod gearbeitet. Die Ergebnisse zeigen auch hier, dass sich bei der Übung von drei verschiedenen berufsbezogenen Aufgaben die Anzahl der richtigen Antworten erhöhte und die damit verbundene Anzahl der Aufforderungen durch den Jobcoach verringerte (Van Laarhoven, Johnson, Van Laarhoven-Myers, Grider \& Grider, 2009).

Teilnehmer*innen können somit durch den Einsatz von technischen Assistenzsystemen zu mehr Autonomie und Selbstständigkeit im Arbeitsalltag und gegebenenfalls zu dem Erleben einer größeren Selbstwirksamkeit 
gelangen. Vor dem Hintergrund der UN-BRK und dem Behindertengleichstellungsgesetz liegt eine konsequente Nutzung des technologischen Fortschritts auf der Hand, um einen gleichberechtigten und umfassenden Zugang zu Informations- und Kommunikationstechnologien für Menschen mit IB zu ermöglichen (Bosse, Schluchter \& Zorn, 2019).

Die von uns entwickelte RehaGoal App fokussiert sich auf die Störungen der exekutiven Funktion, die eine zentrale Rolle bei der erfolgreichen Bewältigung des Alltags und für die berufliche Teilhabe spielt (Müller, 2013; Müller, Ertas, Aust, Gabel \& Schiering, 2019). Zur Therapie
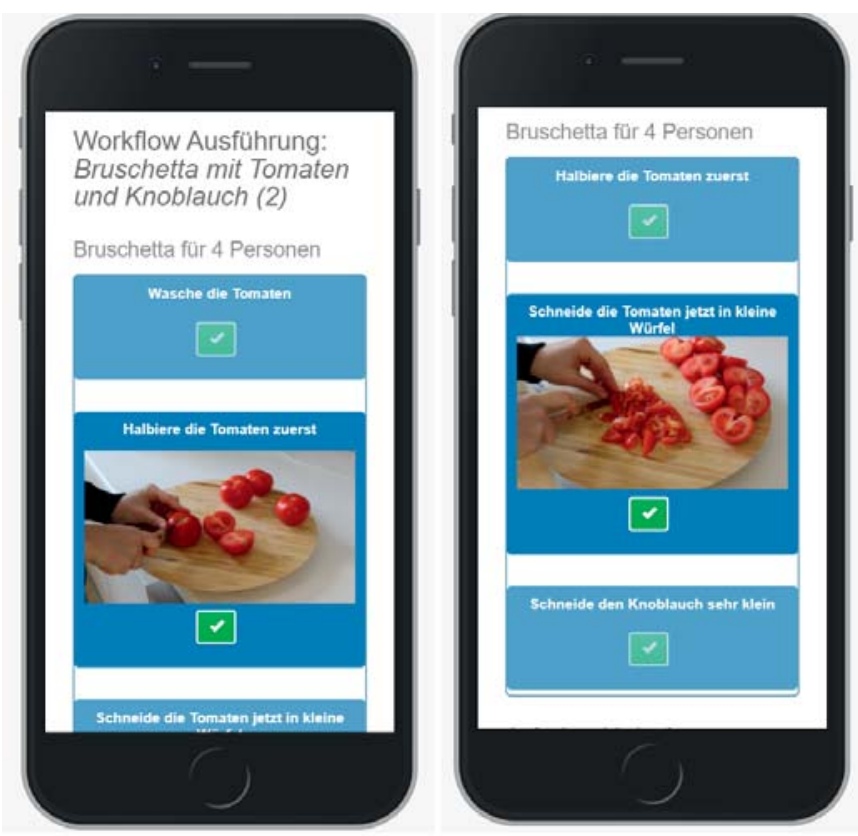

Abbildung 1. Ansicht für RehaGoal App der Teilnehmer*innen. der exekutiven Funktion existieren verschiedene Ansätze, von denen das Goal Management Training (GMT) von Levine et al. (2000) gut untersucht und evidenzbasiert ist (Krasny-Pacini, Chevignard \& Evans, 2014). Das GMT basiert auf der Theorie des Goal Neglects (Duncan, Emslie, Williams, Johnson \& Freer, 1996), also einer Zielvernachlässigung. Zielgruppe sind Personen, die bei komplexen Planungsaufgaben den roten Faden verlieren, stark ablenkbar sind und zu chaotischem Verhalten neigen. Daher steht beim GMT die Zerlegung eines übergeordneten Ziels in Teilziele und überschaubare Schritte zu deren Erreichung im Vordergrund (Levine et al., 2000; Levine et al., 2007).

Handlungen können individuell in Teilziele und einzelne Handlungsschritte untergliedert werden, die anschließend kleinschrittig abgearbeitet werden können. Mithilfe der RehaGoal App ist genau dies jetzt digital möglich. Die RehaGoal App führt schrittweise durch alltägliche und berufliche Abläufe, wie zum Beispiel Kochen nach Rezept (siehe Abbildung 1).

Ziel der RehaGoal App ist die Erlangung größtmöglicher Selbstständigkeit und Autonomie bei den Betroffenen. Zentral für den erfolgreichen Einsatz der RehaGoal App ist die Identifikation relevanter Aufgaben. Die Aufgaben identifizieren Jobcoaches gemeinsam mit Teilnehmer*innen und setzen diese in individuelle Handlungsabläufe, sogenannte Workflows, um (siehe Abbildung 2). Die Bausteine, mit denen die Workflows erstellt werden können, und ihre Funktion sind in Tabelle 1 dargestellt.

Im FBE wird die RehaGoal App im Rahmen zweier Forschungsprojekte seit 2 Jahren zur individuellen Unterstützung der Teilnehmer*innen auf dem allgemeinen Arbeitsmarkt sowie im 3-monatigen Eingangsverfahren eingesetzt.
Abbildung 2. Ansicht des Workflow Editors zur Erstellung von Arbeitsabläufen für die RehaGoal App.

\section{Workflow Editor: Bruschetta mit Tomaten und Knoblauch}

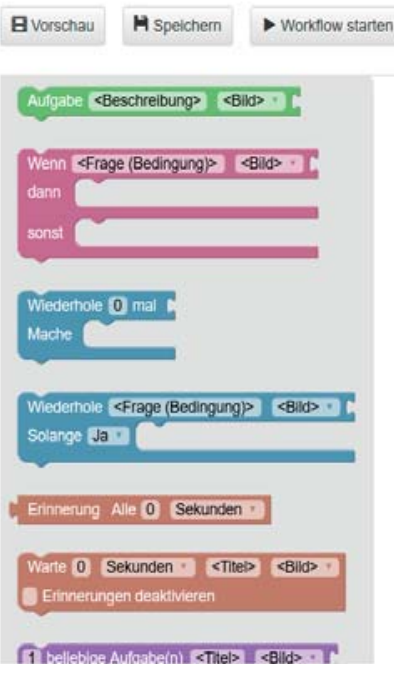

Bilder verwalten

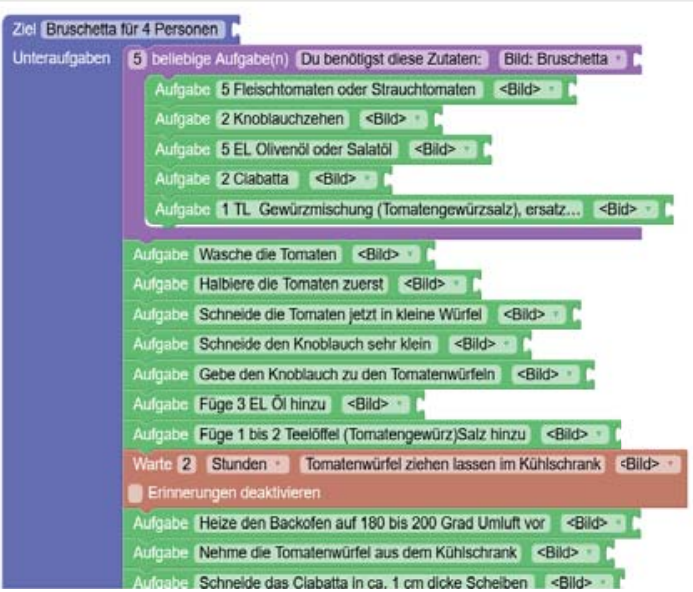


Tabelle 1. Erläuterung der Spezialfunktionen der Workflows der RehaGoal App (modi. Müller et al., 2019)

\begin{tabular}{|c|c|}
\hline Spezialfunktionen & Beschreibung \\
\hline Wiederholungsfunktion & $\begin{array}{l}\text { Sie dient dazu, einen Handlungsschritt x-Mal zu wiederholen oder bis ein definierter Endzustand erreicht } \\
\text { wird. }\end{array}$ \\
\hline Wartefunktion & Mit dieser Funktion kann innerhalb einer Handlungsabfolge für eine ausgewählte Zeit gewartet werden. \\
\hline Erinnerungsfunktion & $\begin{array}{l}\text { Sie soll an einen bestimmten Handlungsschritt erinnern. Diese Erinnerungen können in beliebiger } \\
\text { Frequenz erfolgen. }\end{array}$ \\
\hline Wenn-Dann-Sonst-Funktion & $\begin{array}{l}\text { Sie stellt einen Entscheidungsbaum dar. In Abhängigkeit vom Vorhandensein einer definierten Bedingung } \\
\text { werden unterschiedliche Anweisungen gegeben. }\end{array}$ \\
\hline $\begin{array}{l}\text { Mehrere Handlungsschritte in } \\
\text { beliebiger Reihenfolge }\end{array}$ & $\begin{array}{l}\text { Mit diesem Baustein können mehrere Anweisungen in beliebiger Reihenfolge aufgelistet werden, die zur } \\
\text { Erreichung eines Ziels erfüllt sein müssen. }\end{array}$ \\
\hline Ablaufplanung & $\begin{array}{l}\text { Mithilfe der Ablaufplanung können verschiedene Workflows hintereinander bereitgestellt werden, welche } \\
\text { nacheinander durchgeführt werden können. }\end{array}$ \\
\hline Kalenderfunktion & $\begin{array}{l}\text { Mit der Kalenderfunktion ist es möglich, Workflows zu einem bestimmten Zeitpunkt zu planen und } \\
\text { starten zu lassen. }\end{array}$ \\
\hline
\end{tabular}

\section{Methode}

\section{Teilnehmerin und Teilnehmer}

Für die Zeit im Work-at-Home während des Lockdowns wurden drei Teilnehmer*innen des FBE mit exekutiven Dysfunktionen ausgewählt. Weitere Einschlusskriterien waren Technikkompetenz, grundlegende Selbstständigkeit sowie Sprachverständnis und -produktion.

Es wurden eine Teilnehmerin und zwei Teilnehmer im Alter von 20, 21 und 22 Jahren für diese Pilotstudie identifiziert. Alle Teilnehmer*innen lebten zu diesem Zeitpunkt bei ihren Angehörigen. Eine Kurzbeschreibung der Teilnehmer*innen basierend auf der standardmäßig durchgeführten Anamnese ist in den Tabellen 2 bis 4 dargestellt.

Des Weiteren erfolgte eine neuropsychologische Testung zum planerischen Denken, der Unterdrückung von nichtadäquaten Reaktionen und des Arbeitsgedächtnisses. Außerdem erhielten die Teilnehmer*innen und ihre jeweiligen Jobcoaches einen Fragebogen zur Erfassung exekutiver Dysfunktion (siehe Tabelle 5).

Um die Teilnehmer*innen hinsichtlich ihrer beruflichen Kompetenzen einzuschätzen, führt der FBE standardmäßig die Kompetenzfeststellungsverfahren hamet 2 und hamet e durch. Die Ergebnisse sind in Tabelle 6 zusammengefasst.

\section{Durchführung}

Die Teilnehmer*innen erklärten sich bereit, innerhalb eines Zeitraumes von 3 Wochen für ihre Familien mit der RehaGoal App zu kochen. Die Teilnehmer*innen durften die zu kochenden Gerichte selbst auswählen, um ihre Motivation zu steigern. Sie bekamen den Auftrag, Speisepläne für eine Woche in Tabellenform mit Links zu den Rezepten im Internet zu erstellen. Sie konnten völlig frei entscheiden, was sie kochen wollten und wie anspruchsvoll das Rezept sein sollte.

Nach den ausgewählten Speiseplänen der drei Teilnehmer*innen erstellten die Jobcoaches individuelle Workflows (siehe Abbildung 1). Die fertigen Workflows wurden auf die vom FBE zur Verfügung gestellten Smartphones übertragen und unter Einhaltung der AHA-Regeln den Teilnehmer*innen persönlich übergeben. Die Übertragung der Workflows auf das Smartphone wurde durch den Jobcoach per Telefon und/oder einer Fernwartungssoftware kleinschrittig begleitet.

\section{Evaluation}

Die Evaluation erfolgte mittels eines teilstandardisierten Fragebogens (basierend auf der System Usability Scale von Brooke, 1986), welcher die Erfahrungen und die Nutzerfreundlichkeit der App erfasste. Die drei Teilnehmer*innen nahmen nach dem Kochen an einer telefonischen Nachbefragung teil. Weiterhin führten die Teilnehmer*innen während der 3 Wochen ein Tagebuch. In diesem sollten sie das jeweilige Gericht benennen, positive und negative Erfahrungen festhalten und die benötigte Zeit protokollieren. Zusätzlich wurden die Angehörigen schriftlich befragt. 
Tabelle 2. Kompetenzen und Defizite des Teilnehmers 1 (21 Jahre, männlich)

\begin{tabular}{ll}
\hline Diagnose & Aufmerksamkeitsdefizit-/Hyperaktivitätsstörung und Autismus mit einem Grad der Behinderung von 50 \\
\hline Beobachtete Defizite durch & - Erhöhte Ablenkbarkeit \\
den Jobcoach & - Schwierigkeiten in der selbstständigen Handlungsplanung bei komplexen Aufgaben \\
& - Eingeschränkte Fähigkeit zum Multitasking \\
& - Mangelnde Flexibilität \\
& - ausgeprägtes Kommunikationsbedürfnis in unpassenden Situationen \\
& - Dyskalkulie \\
& - Mangelnde Kritikfähigkeit \\
& - Motiviert, zuverlässig und pünktlich \\
& - Adäquates Sozialverhalten \\
& - Gute Kommunikationsfähigkeit \\
Beobachtete Kompetenzen & - Selbstständige Durchführung von einfachen Aufgaben \\
durch den Jobcoach & - Bereits erlernte komplexe Arbeiten können selbstständig durchgeführt werden \\
& - Gute Gedächtnisleistung \\
\hline
\end{tabular}

Tabelle 3. Kompetenzen und Defizite der Teilnehmerin 2 (20 Jahre, weiblich)

\begin{tabular}{ll}
\hline Diagnose & Lernbehinderung mit einem Grad der Behinderung von 50 \\
\hline Beobachtete Defizite durch & - Schwierigkeiten in der selbstständigen Handlungsplanung bei komplexen Aufgaben \\
den Jobcoach & - Ein selbstständiges Arbeiten anhand von Plänen ist nicht möglich \\
& - Verlangsamung \\
& - Benötigt detaillierte und langsame Erklärungen \\
& - Holt sich nicht oder zu spät Hilfe \\
& - Motiviert, zuverlässig und pünktlich \\
Beobachtete Kompetenzen & - Arbeitet gewissenhaft und konzentriert \\
durch den Jobcoach & - Selbstständige Durchführung von einfachen Aufgaben möglich \\
& - Bereits erlernte Arbeiten können selbstständig durchgeführt werden \\
\hline
\end{tabular}

Tabelle 4. Kompetenzen und Defizite des Teilnehmers 3 (22 Jahre, männlich)

\begin{tabular}{ll}
\hline Diagnose & Trisomie 21 mit einem Grad der Behinderung von 80 \\
\hline Beobachtete Defizite durch & - Impulsives und vorschnelles Handeln \\
den Jobcoach & - Schwierigkeiten in der selbstständigen Handlungsplanung \\
& - Neue Aufgaben müssen oft wiederholt werden \\
& - Eingeschränkte Fähigkeit zum Multitasking \\
& - Defizite im Zeitmanagement \\
& - Gedächtnisdefizite \\
& - Mangelnde Motivation \\
& - Schnelle, verwaschene Sprache \\
& - Mangelnde Flexibilität \\
& - Unrealistische Selbsteinschätzung \\
& - Zuverlässig und pünktlich \\
Beobachtete Kompetenzen & - Kommunikativ \\
durch den Jobcoach & - Bereits erlernte Arbeiten können selbstständig durchgeführt werden \\
\end{tabular}

\section{Ergebnisse}

\section{Teilnehmerbefragung}

Die Teilnehmer*innen gaben an, die RehaGoal App auf einem Smartphone oder Laptop zwischen 1 und 3 Stunden pro Tag zu nutzen. In Abbildung 3 sind die Antworten der drei Teilnehmer*innen auf die Fragen zur Nutzerfreundlichkeit der RehaGoal App dargestellt. Daraus geht hervor, dass die Teilnehmer*innen grundsätzlich sehr gut mit der RehaGoal App zurechtkamen und sie nahezu kein zusätzliches Wissen für die Nutzung erwerben mussten.

In Abbildung 4 sind die Antworten der Teilnehmer*innen zur notwendigen Unterstützung bei der Nutzung der App 
Tabelle 5. Neuropsychologische Ergebnisse der Teilnehmer*innen

\begin{tabular}{|c|c|c|c|}
\hline \multirow{2}{*}{$\begin{array}{l}\text { Neuropsychologisches Leistungsprofil } \\
\text { (Rohwerte) }\end{array}$} & \multicolumn{3}{|c|}{ Teilnehmer*innen } \\
\hline & 1 & 2 & 3 \\
\hline \multicolumn{4}{|l|}{ Planerisches Denken } \\
\hline - Tower of London & 18 & 12 & 12 \\
\hline \multicolumn{4}{|l|}{$\begin{array}{l}\text { Unterdrückung einer nichtadäquaten } \\
\text { Reaktion }\end{array}$} \\
\hline $\begin{array}{l}\text { - Testbatterie zur Aufmerksamkeits- } \\
\text { prüfung Go/NoGo Median (msec) }\end{array}$ & 495 & 518 & 807 \\
\hline $\begin{array}{l}\text { - Testbatterie zur Aufmerksamkeits- } \\
\text { prüfung Go/NoGo Fehler }\end{array}$ & 1 & 0 & 9 \\
\hline $\begin{array}{l}\text { - Testbatterie zur Aufmerksamkeits- } \\
\text { prüfung Go/NoGo Auslassungen }\end{array}$ & 0 & 0 & 18 \\
\hline \multicolumn{4}{|l|}{ Arbeitsgedächtnis } \\
\hline - Zahlenspanne vorwärts & 5 & 7 & 4 \\
\hline - Zahlenspanne rückwärts & 6 & 4 & 4 \\
\hline \multicolumn{4}{|l|}{ Fragebogen } \\
\hline $\begin{array}{l}\text { - Dysexecutive Questionnaire Selbst- } \\
\text { einschätzung }\end{array}$ & 23 & 17 & 48 \\
\hline $\begin{array}{l}\text { - Dysexecutive Questionnaire Fremd- } \\
\text { einschätzung }\end{array}$ & 30 & 21 & 48 \\
\hline
\end{tabular}

Tabelle 6. Hamet-Ergebnisse der Teilnehmer*innen

\begin{tabular}{lccc}
\hline hamet 2/e Berufliche Basiskompetenzen & \multicolumn{3}{c}{ Teilnehmer*innen } \\
\cline { 2 - 4 } & $1^{\circ}$ & $2^{\circ}$ & $3^{*}$ \\
\hline A Routine und Tempo & 4.2 & 2.4 & $28 \%$ \\
$\begin{array}{l}\text { B Werkzeugeinsatz und -steuerung } \\
\text { (einfach) }\end{array}$ & 7.7 & 5.3 & $20 \%$ \\
C Wahrnehmung und Symmetrie & 2.0 & 2.0 & $46 \%$ \\
$\begin{array}{l}\text { D Instruktionsverständnis und -umsetzung } \\
\text { E Werkzeugeinsatz und -steuerung }\end{array}$ & 5.5 & - & $72 \%$ \\
(komplex) & 3.0 & - & $61 \%$ \\
F Messgenauigkeit und Präzision & 5.7 & & \\
\hline
\end{tabular}

Anmerkungen. ${ }^{\circ}=$ Einsatz von hamet 2 , Normwerte (Berufsschule) in Stanine: $1-2=$ unterdurchschnittlich, $3-4=$ unterer Durchschnittsbereich, $5=$ durchschnittlich, 6-7 = oberer Durchschnittsbereich, $8-9=$ überdurchschnittlich; * = Einsatz von hamet e, Werte konnten nur in \% ermittelt werden; - = Der Test konnte nicht durchgeführt werden, weil die Teilnehmerin die Instruktionen nicht verstanden hat.

aufgelistet. Nur der dritte Teilnehmer benötigte bei der Durchführung der Workflows und beim Erledigen der Teilschritte manchmal Hilfe. Bei allen anderen Punkten antworteten die Teilnehmer*innen entweder mit „nein“ oder, wenn sie die Funktion nicht angewendet hatten, mit "nicht genutzt".

In Abbildung 5 sind die Antworten der Teilnehmer* innen zu möglichen Fehlerquellen bei der Nutzung der RehaGoal App aufgelistet. Zwei Personen gaben an, unabsichtlich Teilschritte übersprungen zu haben. Teilnehmer 3 antwortete, manchmal bei Nutzung der App die Erledigung eines Teilschrittes nicht bestätigt zu haben.

Des Weiteren wurden die Teilnehmer*innen auf einer vierstufigen Skala („sehr zufrieden“, „eher zufrieden“, „eher unzufrieden“, „sehr unzufrieden“) gefragt, wie zufrieden sie mit der App insgesamt waren. Zwei Personen antworteten mit „sehr zufrieden“ und eine mit „eher zufrieden".

Am Ende des Fragebogens wurden die Teilnehmer${ }^{*}$ innen nach positiven und negativen Erfahrungen befragt und ob sie noch Anregungen, Kritik oder Wünsche bezüglich der App hätten.

Der erste Teilnehmer äußerte sich durchweg positiv. Die zweite Teilnehmerin sagte, dass sie die Sprachausgabe und die Untergliederung in einzelne Handlungsschritte nützlich gefunden hätte. Dem dritten Teilnehmer gefiel, dass alle Zutaten in der App übersichtlich dargestellt und ihm die Schritte aufgezeigt wurden. Dadurch konnte er neues Wissen über das Kochen erwerben. Die Frage, ob ihnen etwas bei der Nutzung der RehaGoal App nicht gut gefallen habe, wurde verneint. Ein Teilnehmer äußerte den Wunsch, während des Wartens in der Wartefunktion gerne weitere Aufgaben erledigen zu können.

\section{Tagebuchauswertung}

Der erste Teilnehmer kochte neun Rezepte und benötigte dafür zwischen 45 und 150 Minuten. Auf die Frage, was bei der Durchführung gut verlaufen sei, berichtete er, dass alles gut verlaufe. Einmal notierte er, dass er es toll fand, vor dem Beginn des Kochens alle benötigten Zutaten rauszusuchen.

Die zweite Teilnehmerin kochte 14 verschiedene Rezepte mit der App und benötigte hierfür zwischen 25 und 60 Minuten. Die Teilnehmerin nutzte für die ersten zwei Rezepte ein Smartphone ohne Verwendung der Sprachausgabe. Laut Tagebuch hatte sie Schwierigkeiten mit dem Lesen und verwechselte deshalb die Reihenfolge mehrerer Schritte. Daher sollte die Sprachausgabe zum Einsatz kommen, um Lesefehler zu vermeiden. Die Teilnehmerin hielt fest, dass das Kochen gut verlaufe, da die einzelnen Handlungsschritte verständlich erklärt würden. Kritisiert wurde, dass die Wartefunktion einmal zu kurz eingestellt war.

Der dritte Teilnehmer kochte insgesamt achtmal für seine Familie und benötigte dafür zwischen 15 und 60 Minuten. Auf die Frage „Was lief gut?“ antwortete der Teilnehmer knapp „gut“. Beanstandet wurde von ihm allerdings, dass eine parallele Bearbeitung von zwei Speisen nicht möglich sei und er somit erst die Hauptspeise beenden musste und dann im Anschluss mit der Beilage beginnen konnte oder andersherum. 
Abbildung 3. Ergebnisse der teilstandardisierten Fragen zur RehaGoal App - Allgemeiner Teil.

\begin{tabular}{|c|c|c|c|c|c|}
\hline Items & $\mathbf{J a}$ & Manchmal & Nein & Vielleicht & $\begin{array}{c}\text { Weiß ich } \\
\text { nicht }\end{array}$ \\
\hline $\begin{array}{l}\text { Fühlen Sie sich generell fit im Umgang mit } \\
\text { Smartphones/Tablets/Laptops? }\end{array}$ & & & & & \\
\hline Ist die App einfach zu benutzen? & & & & & \\
\hline Konnten Sie mit der App alleine kochen? & & & & & \\
\hline Fühlen Sie sich sicher bei der Nutzung der App? & & & & & \\
\hline $\begin{array}{l}\text { Hat Sie die App in der Corona-Zeit bei Ihrem } \\
\text { Arbeitsauftrag „Kochen für die Familie“ unterstut }\end{array}$ & & & & & \\
\hline Hat die App Ihren Alltag erleichtert? & & & & & \\
\hline $\begin{array}{l}\text { Mussten Sie viel lernen, bevor Sie mit der App } \\
\text { zurechtkamen? }\end{array}$ & & & & & \\
\hline Würden Sie die App in Zukunft häufiger nutzen? & & & & 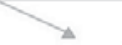 & \\
\hline
\end{tabular}

Haben Sie bei den folgenden Punkten

Ja Manchmal Nein

Nicht Unterstützung benötigt? genutzt

Bei der Durchführung der Workflows

Beim Erledigen der Teilschritte

Beim Kopieren der Workflows auf das Handy

Bei der Nutzung der Smartwatch

Bei der Einstellung der Sprachausgabe

Beim Anzeigen von Bildern

Bei weiteren technischen Problemen

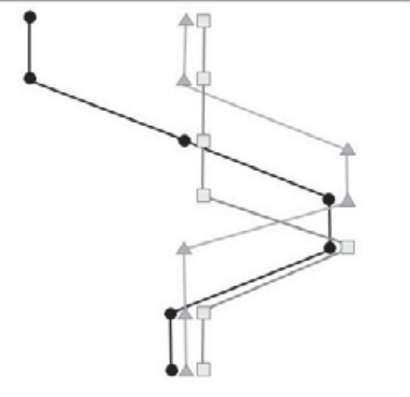

Note. $\square=$ Teilnehmer $1, \Delta=$ Teilnehmerin $2, \bullet=$ Teilnehmer 3
Abbildung 5. Potenzielle Fehlerquellen bei Nutzung der RehaGoal App.
Abbildung 4. Benötigte der RehaGoal App.

\section{Angehörigenbefragung}

Nach der Nutzung der RehaGoal App erhielten die Angehörigen der Teilnehmer*innen einen kurzen, sechs Fragen umfassenden Fragebogen (Abbildung 6).

Die Angehörigen gaben größtenteils an, dass ihre Kinder zufriedenstellend mit der App kochen konnten. Des Weiteren gaben zwei Angehörige an, dass ihr Kind wäh- rend des Kochens zusätzliche Unterstützung benötigt habe, und ein Angehöriger gab an, zum Teil unterstützt zu haben. Als Grund für die Unterstützung wurde angegeben, dass der Teilnehmer hektisch geworden sei und den Überblick verloren habe. Des Weiteren wurde erfragt, ob die App für künftige häusliche Tätigkeiten von ihren Kindern zur Unterstützung genutzt werden könnte. Hier antwortete einer mit ,ja“ und zwei mit „teilweise“. Als eine weitere 


\begin{tabular}{l}
\hline Items \\
\hline Mein Kind konnte mit der App die Aufgaben „Kochen für die Familie“ \\
zufriedenstellend durchführen \\
Mein Kind benötigt zusätzliche Hilfe von mir, während der Nutzung der App \\
Durch die Nutzung der App, ist mein Kind selbstständiger beim Kochen \\
Die Aufgabe „Kochen für die Familie“ war eine sinnstiftende Aufgabe für mein \\
Kind. \\
Die Aufgabe „Kochen für die Familie“ war eine arbeitsbezogene Aufgabe für mein \\
Kind. \\
Die Aufgabenerledigung mit der App verlief einfacher als die Erledigung der \\
Aufgaben, die mein Kind zuvor vom FBE (z.B. Klimaretter, Industrialisierung) \\
bekommen hat. \\
Ich könnte mir vorstellen, dass mein Kind die App auch nach der Corona-Phase im \\
Alltag bei uns Zuhause einsetzen kann. \\
\hline Note. $\square=$ Teilnehmer 1, $1=$ Teilnehmerin 2, $\bullet=$ Teilnehmer 3
\end{tabular}

Abbildung 6. Allgemeine Fragen zur RehaGoal App - Angehörigenbefragung.
Einsatzmöglichkeit wurden routinemäßige Hausarbeiten genannt, wie zum Beispiel Wäsche waschen, Bett machen, Zimmer aufräumen etc.

Auf die Frage, ob sich durch die Nutzung der App das Kochen nach Rezept für sie als Elternteil verändert habe, antworteten zwei Angehörige mit, es sei ,gleichbleibend geblieben“ und ein Angehöriger antwortete, dass es , anstrengender geworden" sei.

Die Angehörigen wurden ebenfalls nach positiven und negativen Eindrücken von der RehaGaol App sowie nach Anregungen, Kritik oder Wünschen gefragt.

Die Angehörigen fanden die App sehr übersichtlich gestaltet. Zudem gaben sie an, die klare Abfolge der Handlungsschritte gut zu finden, und berichteten, dass durch die Anwendung der App die Motivation zum Kochen bei ihren Kindern gestiegen sei.

Kritisiert wurden Details, wie zum Beispiel die fehlende Möglichkeit, die Anzahl der Portionen im Nachhinein zu ändern, und dass durch die Wartefunktion die App als zeitaufwendig wahrgenommen wurde, da während des Wartens keine weiteren Zutaten vorbereitet werden konnten.

Die Angehörigen wünschten sich eine Zurücktaste, um die vorherigen Handlungsschritte in der App zu sehen, und in der Wartefunktion die Möglichkeit, den Timer wegklicken bzw. währenddessen weiterarbeiten zu können.

\section{Diskussion}

In dieser multiplen Einzelfallstudie mit drei Teilnehmer*innen wurde die RehaGoal App während der CoronaPandemie bei Menschen mit IB zum Kochen eingesetzt und erprobt. Hierbei wurden die Teilnehmer*innen schrittweise gemäß der Theorie des GMT (Levine et al., 2007; Müller et al., 2019) durch das Rezept geführt.
Der erste Teilnehmer (Diagnose: Autismus und Aufmerksamkeitsdefizit-/Hyperaktivitätsstörung) hat laut Anamnese Schwierigkeiten in seiner Aufmerksamkeit und Handlungsplanung. Seine Kompetenzen zeigten sich bei der selbstständigen Durchführung von bereits erlernten Arbeiten. In den neuropsychologischen Testungen zeigte er Defizite im Arbeitsgedächtnis und im hamet 2 unterdurchschnittliche Leistung in Routine- und Tempoaufgaben, bei der Wahrnehmung und Symmetrie sowie bei Werkzeugeinsatz und -steuerung (komplex). Die Arbeit mit der RehaGoal App beim Kochen bewertete er als durchweg positiv und würde sie gern weiterhin verwenden. Der Angehörige des ersten Teilnehmers berichtete, dass dieser teilweise zusätzliche Hilfestellung während des Kochens benötigt habe und die Aufgabe auch nur teilweise zufriedenstellend habe durchführen können.

Der zweiten Teilnehmerin (Diagnose: Lernbehinderung) fällt es laut Anamnese schwer, anhand von Plänen selbstständig zu arbeiten. Zudem benötigt sie bei der Bearbeitung von komplexen Aufgaben oft detaillierte und langsame Erklärungen. Bereits erlernte Aufgaben kann sie selbstständig durchführen. In der neuropsychologischen Diagnostik zeigte sie ebenfalls Defizite im planerischen Denken und im Arbeitsgedächtnis. Im hamet 2 erreichte sie bei Werkzeugeinsatz und -steuerung (einfach) eine durchschnittliche Leistung, bei allen anderen Untertests schloss sie unterdurchschnittlich ab. Laut Fragebogen und Tagebuch konnte sie mit der App durch die Sprachausgabe alleine kochen und fühlte sich sicher bei ihrer Nutzung. Offen bleibt, ob sie die App auch in Zukunft tatsächlich häufiger nutzen möchte. Ihr Angehöriger berichtete, dass sie teilweise Unterstützung beim Kochen benötigt habe, die Aufgaben jedoch zufriedenstellend habe durchführen können.

Der dritte Teilnehmer (diagnostiziert mit Trisomie 21) zeigt laut Anamnese Defizite in der selbstständigen Handlungsplanung und handelt impulsiv und vorschnell bei der 
Aufgabendurchführung. In den neuropsychologischen Tests zeigte er die schlechtesten Ergebnisse. Besonders beeinträchtigt ist er in der Unterdrückung von nichtadäquaten Reaktionen. Beim hamet e fielen ihm die Routine- und Tempoaufgaben sowie Werkzeugeinsatz und -steuerung (einfach) schwer. Bei allen anderen Aufgaben erreichte er ca. die Hälfte der Punkte. Die Nutzung der RehaGoal App empfand der Teilnehmer als positiv und würde sie in Zukunft nutzen. So berichtete er außerdem, dass die App für ihn einfach zu bedienen gewesen wäre und diese ihn beim Kochen auch unterstützt habe. Der Angehörige von Teilnehmer 3 berichtete, dass dieser das Kochen für die Familie zufriedenstellend habe durchführen können, allerdings zusätzliche Hilfe bei der Durchführung des Kochens benötigt habe. Obwohl der Angehörige als Einziger angab, dass das Kochen anstrengender geworden sei, antwortete dieser, dass er sich vorstellen könnte, auch nach der Corona-Pandemie die App weiter zu nutzen.

Bei näherem Vergleich der Angaben zwischen den Teilnehmer*innen und deren Angehörigen fällt eine Diskrepanz auf. Nur ein Teilnehmer berichtete, manchmal bei der Durchführung und der Erledigung der Teilschritte Unterstützung benötigt zu haben. Die Angehörigen allerdings berichteten alle, dass sie bei ihren Kindern teilweise oder oft geholfen hätten. Auf die Frage, warum die Kinder zusätzliche Hilfe gebraucht hätten, beschrieb nur ein Angehöriger, dass sein Kind hektisch geworden sei. Bei den anderen beiden gab es dazu keine näheren Informationen. Um die Workflows noch besser an die Teilnehmerinnen anzupassen, wäre es hilfreich, mehr über die Art der Unterstützung durch die Angehörigen zu erfahren. Des Weiteren berichtete die zweite Teilnehmerin, keine Unterstützung von den Jobcoaches bei der Einstellung der Sprachausgabe und dem Kopieren der Workflows benötigt zu haben.

Zwei Angehörige berichteten, dass sich die Motivation zum Kochen bei ihren Kindern gesteigert habe. Eine mögliche Ursache für die Motivationssteigerung könnte ein größeres Autonomieerleben der Teilnehmerinnen sein. So wurde in der Studie von Pelletier und Joussemet (Emond Pelletier \& Joussemet, 2017) herausgefunden, dass das erhöhte Autonomieerleben zu größerer Zufriedenheit führt und dadurch der Aktivität mehr Wert beigemessen wird.

Grundsätzlich stellt das Kochen eine komplexe, mehrschrittige Abfolge von verschiedenen Handlungen dar, die sich gut zur Beobachtung und Verbesserung der Defizite in der exekutiven Funktion eignet. So führte zum Beispiel das GMT bei erwachsenen Menschen mit erworbenen Hirnschädigungen beim Kochen zu weniger Fehlern (Levine et al., 2000). Krasny-Pacini et al. (2014) untersuchten den Einfluss des GMT-Einsatzes auf das Kochen bei hirnverletzten Kindern. Es zeigte sich, dass sich nur zwei der fünf Kinder beim Kochen verbesserten. Möglicherweise könnten Menschen mit angeborenen Hirnschädigungen von der RehaGoal App als Kompensationsmittel stärker profitieren. Die vorliegende Pilotstudie zeigte, dass die Angehörigen ihre Kinder trotz der Strukturierung durch die App zusätzlich unterstützen mussten. Dies könnte darauf hindeuten, dass die Workflows nicht genügend selbsterklärend bzw. einfach verfasst wurden. Eine weitere Erklärung wäre, dass nur einige Aspekte der exekutiven Funktion mit der App kompensiert werden können, wie zum Beispiel die Handlungsplanung und -durchführung, jedoch nicht die kognitive Flexibilität, das Arbeitsgedächtnis, die Selbstregulation und die Unterdrückung einer nichtadäquaten Reaktion, welche ebenfalls zu den Defiziten der Teilnehmer*innen gehörten (siehe Tabelle 2).

Aufgrund des gegenwärtigen Mangels an Diagnostikmitteln bei Menschen mit IB (García-Alba et al., 2017) sind die in dieser Studie verwendeten neuropsychologischen Parameter für die Allgemeinbevölkerung normiert und wurden daher mit den Ergebnissen des Kompetenzfeststellungsverfahrens hamet e und hamet 2 sowie zusätzlichen Beobachtungen durch die Jobcoaches ergänzt. Alle Teilnehmer*innen zeigten in allen Erhebungsformen unter anderem Schwierigkeiten in ihrer exekutiven Funktion. Memisevic und Sinanovic (2013) stellten in ihrer Studie fest, dass die exekutiven Funktionen umso stärker beeinträchtigt sind, je ausgeprägter die IB ist. Obwohl Teilnehmer 3 den höchsten Grad der Behinderung hatte und am schlechtesten bei der Diagnostik abschnitt, gelang es ihm laut des Angehörigen, mit der App zufriedenstellend zu kochen.

Hinsichtlich der Spezialfunktionen (siehe Tabelle 1) der RehaGoal App wurde von der zweiten Teilnehmerin die Sprachausgabe als hilfreich eingestuft, da diese dazu geführt habe, dass sie sich besser auf die Aufgabe konzentrieren konnte und nicht noch zusätzlich durch das Lesen abgelenkt war. Die Wartefunktion wurde von den Teilnehmer*innen und deren Angehörigen kritisiert, da in der Wartezeit keine weitere Aufgabe erledigt und der Timer nicht nachjustiert werden konnte.

Insgesamt zeigte sich in der Pilotstudie während des Lockdowns der Corona-Pandemie der Mehrwert der RehaGoal App. In einer Zeit, als die Jobcoaches ihre Teilnehmer*innen nicht persönlich besuchen und anleiten konnten, war es möglich, mithilfe der RehaGoal App eine komplexe und mehrschrittige Tätigkeit wie das Kochen durchzuführen und dadurch das Autonomieerleben der Teilnehmer*innen zu steigern. Alle Teilnehmer*innen berichteten, dass sie sich sicher im Umgang mit der RehaGoal App gefühlt hätten und beim Kochen durch diese unterstützt worden seien. Insgesamt sind die Teilnehmer*innen mit der App zufrieden und zwei von ihnen planen, die RehaGoal App in Zukunft zu verwenden. 


\section{Literatur}

Beauftragter der Bundesregierung für die Belange von Menschen mit Behinderungen. (Hrsg.). (2018). Die UN-Behindertenrechtskommission. Übereinkommen über die Rechte von Menschen mit Behinderungen. Verfügbar unter https://www.bmas.de/DE/ Service/Medien/Publikationen/a729-un-konvention.html

Bosse, I., Schluchter, J.-R. \& Zorn, I. (Hrsg.). (2019). Handbuch Inklusion und Medienbildung. Weinheim: Beltz.

Brooke, J. (1986). SUS - A quick and dirty usability scale. Verfügbar unter http://www.usabilitynet.org/trump/documents/Suschapt.doc

Bundesarbeitsgemeinschaft der überörtlichen Träger der Sozialhilfe/con_sens (2019, 15. März). Kennzahlenvergleich Eingliederungshilfe der überörtlichen Träger der Sozialhilfe - 2017. Verfügbar unter http://kennzahlenvergleich-eingliederungshilfe. de/images/berichte/2019-04-08\%20BAGS\%20Bericht\%20 2017_barrierefrei_final.pdf

Bundesarbeitsgemeinschaft Werkstätten für behinderte Menschen. (2019, 16. Mai). Sinkende Zugangszahlen im Eingangsverfahren und Berufsbildungsbereich. Verfügbar unter https:// www.bagwfbm.de/article/3976

Burckley, E., Tincani, M. \& Guld Fisher, A. (2014). An iPad'TM-based picture and video activity schedule increases community shopping skills of a young adult with autism spectrum disorder and intellectual disability. Developmental Neurorehabilitation, 18, 131-136. https://doi.org/10.3109/17518423.2014.945045

Cannella-Malone, H., Sigafoos, J., O’Reilly, M., de la Cruz, B., Edrisinha, C. \& Lancioni, G.E. (2006). Comparing video prompting to video modeling for teaching daily living skills to six adults with developmental disabilities. Education and Training in Developmental Disabilities, 41, 344-356. https://doi.org/10.1177\%2F1 088357610380412

Deutsches Institut für Menschenrechte e.V. (Hrsg.). (2019). Wer Inklusion will, sucht Wege. Zehn Jahre UN-Behindertenrechtskonvention in Deutschland. Verfügbar unter https://www.institutfuer-menschenrechte.de/fileadmin/user_upload/Publika tionen/ANALYSE/Wer_Inklusion_will_sucht_Wege_Zehn_Jahre_ UN_BRK_in_Deutschland.pdf

Duncan, J., Emslie, H., Williams, P., Johnson, R. \& Freer, C. (1996). Intelligence and the frontal lobe: The organization of goal-directed behavior. Cognitive Psychology, 30, 257-303. https://doi. org/https://doi.org/10.1006/cogp.1996.0008

Emond Pelletier, J. \& Joussemet, M. (2017). The benefits of supporting the autonomy of individuals with mild intellectual disabilities: An experimental study. Journal of Applied Research in Intellectual Disabilities, 30, 830-846. https://doi.org/https://doi. org/10.1111/jar.12274

García-Alba, J., Esteba-Castillo, S., Castellanos López, M.Á., Rodríguez Hidalgo, E., Ribas Vidal, N., Moldenhauer Díaz, F. et al. (2017). Validation and normalization of the Tower of LondonDrexel University Test 2nd Edition in an adult population with intellectual disability. Spanish Journal of Psychology, 20, E32, 1-14. https://doi.org/https://doi.org/10.1017/sjp.2017.30

Garon, N., Bryson, S. E. \& Smith, I. M. (2008). Executive function in preschoolers: A review using an integrative framework. Psychological Bulletin, 134, 31-60. https://doi.org/10.1037/0033-29 09.134.1.31

Krasny-Pacini, A., Chevignard, M. \& Evans, J. (2014). Goal Management Training for rehabilitation of executive functions: A systematic review of effectiveness in patients with acquired brain injury. Disability and Rehabilitation, 36, 105-116. https://doi.org /10.3109/09638288.2013.777807

Levine, B., Robertson, I. H., Clare, L., Carter, G., Hong, J., Wilson, B.A. et al. (2000). Rehabilitation of executive functioning: An experimental-clinical validation of goal management training. Jour- nal of the International Neuropsychological Society, 6, 299-312. https://doi.org/10.1017/s1355617700633052

Levine, B., Stuss, D.T., Winocur, G., Binns, M.A., Fahy, L., Mandic, M. et al. (2007). Cognitive rehabilitation in the elderly: Effects on strategic behavior in relation to goal management. Journal of the International Neuropsychological Society, 13, 143-152. https://doi.org/10.1017/s1355617707070178

Mahone, E.M. \& Hoffman, J. (2007). Behavior ratings of executive function among preschoolers with ADHD. Clinical Neuropsychologist,21,569-586.https://doi.org/10.1080/13854040600762724

Memisevic, H. \& Sinanovic, O. (2013). Executive function in children with intellectual disability - the effects of sex, level and aetiology of intellectual disability. Journal of Intellectual Disability Research, 58, 830-837. https://doi.org/10.1111/jir.12098

Müller, S.V. (2013). Fortschritte der Neuropsychologie. Störungen der Exekutivfunktionen (10. Aufl.). Göttingen: Hogrefe.

Müller, S. V., Ertas, F., Aust, J., Gabel, A. \& Schiering, I. (2019). Kann eine mobile Anwendung helfen abzuwaschen? Zeitschrift für Neuropsychologie, 30, 123-131. https://doi.org/10.1024/1016264X/a000256

Müller, S.V. \& Klein, T. (2019). Diagnostik und Therapie von exekutiven Dysfunktionen bei neurologischen Erkrankungen, S2e-Leitlinie. In Deutsche Gesellschaft für Neurologie (Hrsg.), Leitlinien für Diagnostik und Therapie in der Neurologie. Verfügbar unter www.dgn.org/leitlinien und https://www.awmf.org/leitlinien/ detail/ll/030-125Rauch, A. (2005). Behinderte Menschen auf dem Arbeitsmarkt. In R. Bieker (Hrsg.), Teilhabe am Arbeitsleben. Wege der beruflichen Integration von Menschen mit Behinderung (S. 25-43). Stuttgart: Kohlhammer.

Sigafoos, J., O'Reilly, M., Cannella, H., Edrisinha, C., de la Cruz, B., Upadhyaya, M. et al. (2007). Evaluation of a video prompting and fading procedure for teaching dish washing skills to adults with developmental disabilities. Journal of Behavioral Education, 16, 93-109. https://doi.org/10.1007/s10864-006-9004-z

Stuss, D.T. (2011). Functions of the frontal lobes: Relation to executive functions. Journal of the International Neuropsychological Society, 17, 759-765. https://doi.org/10.1017/s13556177 11000695

Trost, R. (2013). Integrationsfachdienste. In G. Theunissen, W. Kulig \& K. Schirbort (Hrsg.), Handlexikon Geistige Behinderung. Schlüsselbegriffe aus der Heilpädagogik, Sozialen Arbeit, Medizin, Psychologie, Soziologie und Sozialpolitik (2. Aufl., S. 184186). Stuttgart: Kohlhammer.

Van Laarhoven, T., Johnson, J.W., Van Laarhoven-Myers, T., Grider, K.L. \& Grider, K.M. (2009). The effectiveness of using a video $\mathrm{iPod}^{\mathrm{TM}}$ as a prompting device in employment settings. Journal of Behavioral Education, 18, 119-141. https://doi.org/10.1007/ s10864-009-9077-6

\section{Historie}

Eingereicht und akzeptiert: 6. Januar 2021

\section{Interessenkonflikt}

Alle Autoren geben keinen Interessenkonflikt an.

\section{Förderung}

„Security Referenzmodell Industrie 4.0“ Fördermittelgeber: Niedersächsisches Ministerium für Wissenschaft und Kultur Förderkennzeichen:ZN3224 (https://securin.de/). Open AccessVeröffentlichung ermöglicht durch Ostfalia Hochschule für angewandte Wissenschaften.

\section{Danksagung}

Wir bedanken uns bei allen Teilnehmer*innen und deren Angehörigen, die uns immer bereitwillig Auskunft gaben, sowie beim Team 
des FBE der DRK-inkluzivo Wolfenbüttel gGmbH, insbesondere Nicole Arendt, Uwe Rump-Kahl, Marie Gentz, Franziska Krüger. Abschließend einen großen Dank an die Angehörigen, die das Workat-Home der Teilnehmer*innen maßgeblich mit unterstützten.

\section{Prof. Dr. Sandra Verena Müller}

Ostfalia Hochschule Wolfenbüttel

Fakultät Soziale Arbeit

Salzdahlumer Str. 46/48

38302 Wolfenbüttel

Deutschland

s-v.mueller@ostfalia.de 